\title{
Sharp lower bounds on density for area-minimizing cones
}

\author{
Tom Ilmanen ANd BRIAn White*
}

\begin{abstract}
We prove that the density of a topologically nontrivial, area-minimizing hypercone with an isolated singularity must be greater than $\sqrt{2}$. The Simons' cones show that $\sqrt{2}$ is the best possible constant. If one of the components of the complement of the cone has nontrivial $k^{\text {th }}$ homotopy group, we prove a better bound in terms of $k$; that bound is also best possible. The proofs use mean curvature flow.
\end{abstract}

MSC 2010 SUBJECT CLASSIFICATIONS: Primary 53A10; secondary 49Q05, $53 \mathrm{C} 44$.

KEYWORDS AND PHRASES: Area-minimizing cone, density, mean curvature flow.

\section{Introduction}

In this paper, we prove some sharp lower bounds on densities of areaminimizing hypercones or, equivalently, on volumes of certain closed minimal hypersurfaces in round spheres. We begin by indicating why such density bounds are of interest. Recall that if $M$ is an $m$-dimensional minimal variety in a Riemannian manifold and if $x$ is an interior point of $M$, then the density of $M$ at $x$ is

$$
\Theta(M, x):=\lim _{r \rightarrow 0} \frac{\operatorname{area}(M \cap \mathbf{B}(x, r))}{\omega_{m} r^{m}},
$$

where $\omega_{m}$ is the $m$-dimensional volume of the unit ball in $\mathbf{R}^{m}$. The limit exists by the monotonicity formula. The density is 1 at any multiplicity 1 regular point, and it is strictly greater than 1 at any singular point (by Allard's regularity theorem). If $M$ is a cone with vertex $x$, then the ratio in (1) is independent of $r$; in that case, we write $\Theta(M)=\Theta(M, x)$.

This paper treats the case of area-minimizing hypersurfaces (either integral currents or flat chains mod 2). Consider the following question:

*The research of the second author was supported by the National Science Foundation grants DMS-0707126, DMS-1105330, and DMS-1404282. 
Q1. What is the infimum of $\Theta(M, x)$ among all pairs $(M, x)$ where $M$ is an area minimizing hypersurface (in some Riemannian manifold) and $x$ is an interior singular point of $M$ ?

Here "interior point of $M$ " means "point in the support of $M$ but not in the support of $\partial M "$.

Note that if $x$ is an interior singular point of $M$ and if $C$ is a tangent cone to $M$ at $x$, then $C$ is an area minimizing hypercone in Euclidean space with a singularity at its vertex, and $\Theta(C)=\Theta(M, x)$. Furthermore, standard dimension reducing arguments show that either $C$ has an isolated singularity at its vertex, or else there is another area minimizing hypercone $C^{\prime}$ of lower dimension such that $C^{\prime}$ has an isolated singularity at its vertex and such that $\Theta\left(C^{\prime}\right) \leq \Theta(C)$. Thus the question Q1 is equivalent to:

Q2. What is the infimum of $\Theta(C)$ among all area-minimizing hypercones $C$ such that $C$ has an isolated singularity at the origin?

In this paper, we give a sharp answer to question Q2 provided one restricts the cones $C$ to those that are topologically nontrivial. In particular, we prove:

Theorem 1. Suppose that $C \subset \mathbf{R}^{n}$ is an area-minimizing hypercone with an isolated singularity at the origin. Suppose also that $C$ is topologically nontrivial in the following sense: at least one of the two components of $\mathbf{R}^{n} \backslash C$ is non-contractible. Then the density of $C$ at the origin is greater than $\sqrt{2}$.

If one wants a constant independent of the dimension $n$, then $\sqrt{2}$ is the best possible because the Simons' cone

$$
C_{m, m}:=\left\{(x, y) \in \mathbf{R}^{m} \times \mathbf{R}^{m}=\mathbf{R}^{2 m}:|x|=|y|\right\}
$$

is both topologically nontrivial and area-minimizing for $m \geq 4$, and by a straightforward calculation (see section 4) its density $\Theta\left(C_{m, m}\right)$ tends to $\sqrt{2}$ as $m$ tends to infinity.

Many years ago Bruce Solomon conjectured that the answer to question Q2 above is $\sqrt{2}$. Our work shows that Solomon's conjecture is true in the class of topologically nontrivial cones.

We remark that all of the many known examples (see [Law91]) of areaminimizing hypercones $C$ with isolated singularities are topologically nontrivial. On the other hand, there are examples ([Hsi83a], [Hsi83b], [HS86]) of minimal embeddings of $m$-spheres into $(m+1)$-spheres that are not totally geodesic. The corresponding cones are minimal hypercones with isolated singularities at their vertices and are topologically trivial. However, we do not know whether those cones minimize area.

Theorem 1 can be restated in terms of minimal submanifolds of spheres: 
Theorem $\mathbf{1}^{\prime}$. Let $\Gamma$ be a closed minimal hypersurface in the unit sphere $\partial \mathbf{B} \subset \mathbf{R}^{n}$. Suppose that the corresponding cone

$$
C=\{r x: x \in \Gamma, r \geq 0\}
$$

is area-minimizing. Suppose also that at least one of the components of $(\partial \mathbf{B}) \backslash$ $\Gamma$ is not contractible. Then the area of $\Gamma$ is greater than $\sqrt{2}$ times the area of the totally geodesic $(n-2)$-sphere in $\partial \mathbf{B}$.

To see that the topological hypotheses of the two theorems are equivalent, note that if $U$ is a component of $\mathbf{R}^{n} \backslash C$, then $U \cap \partial \mathbf{B}$ is the corresponding component of $(\partial \mathbf{B}) \backslash \Gamma$. Also, $U$ is homeomorphic to $(U \cap \partial \mathbf{B}) \times \mathbf{R}$, so $U$ is contractible if and only if $U \cap \partial \mathbf{B}$ is contractible.

Let $C$ be a cone as in Theorem 1. Since one of the components of $\mathbf{R}^{n} \backslash C$ is non-contractible, one of its homotopy groups, say the $k^{\text {th }}$ homotopy group, is nontrivial. One can get a better lower bound for $\Theta(C)$ if one allows a constant that depends on $k$. In particular, we show in Theorem 2 that

$$
\Theta(C) \geq d_{k}=\left(\frac{k}{2 \pi e}\right)^{k / 2} \sigma_{k}
$$

where $d_{k}$ is the Gaussian density of a shrinking $k$-dimensional sphere and $\sigma_{k}$ is the area of the unit $k$-dimensional sphere. (Gaussian density plays the role in mean curvature flow that density does is minimal surface theory. See [Whi97].)

As before, this result is sharp: for any $\epsilon>0$, there is an $n$ and a cone $C \subset \mathbf{R}^{n}$ such that $C$ satisfies the hypotheses of the theorem and such that

$$
\Theta(C)<d_{k}+\epsilon .
$$

See section 4 .

There are no singular area-minimizing hypercones of dimension less than 7. In another paper we will show that Theorem 1 continues to hold if the hypothesis that the cone is area-minimizing is replaced by the hypothesis that the cone is minimal and has dimension less than 7 . We will also prove a somewhat weakened version of Theorem 2 for cones of dimension less than 7.

The proofs of the theorems use various facts about mean curvature flow. We describe those facts as we need them. For readers who would prefer to know the relevant facts ahead of time, we have stated most of them in appendix A. However, the proof of theorem 1 requires much less than is stated there. In particular, for theorem 1, it suffices to consider mean curvature flow up to the first singular time. 


\section{The proof of Theorem 1}

Proof of theorem 1. Let $M$ be any minimal variety in $\mathbf{R}^{n}$. Then $M$ is in equilibrium for mean curvature flow. That is,

$$
\mathcal{M}: t \in \mathbf{R} \mapsto M
$$

is a mean curvature flow. Note that $(x, t)$ is a singular point of $\mathcal{M}$ if and only if $x$ is a singular point of $M$. Furthermore, the Gaussian density of $\mathcal{M}$ at $(x, t)$ is equal to the density of $M$ at $x$ :

$$
\Theta(\mathcal{M},(x, t))=\Theta(M, x) .
$$

(The definition and basic properties of Gaussian density may be found in [Whi97], for example.)

Recall the following upper semicontinuity property of Gaussian density (which follows fairly easily from Huisken's monotonicity of density): if $\mathcal{M}_{i}$ is a sequence of mean curvature flows that converge (as Brakke flows) to $\mathcal{M}$ and if $X_{i}$ is a sequence of points in spacetime that converge to $X$, then

$$
\Theta(\mathcal{M}, X) \geq \limsup \Theta\left(\mathcal{M}_{i}, X_{i}\right) .
$$

Now let $M$ be the portion of the cone $C$ in the unit ball $\mathbf{B}$ centered at the origin: $M=C \cap \mathbf{B}$. As above, we let $\mathcal{M}$ be the static mean curvature flow (2). The singular points of $\mathcal{M}$ are precisely the points $(0, t), t \in \mathbf{R}$.

We will construct for each sufficiently small $\epsilon>0$ a mean curvature flow

$$
\mathcal{M}_{\epsilon}: t \in \mathbf{R} \mapsto M_{\epsilon}(t)
$$

with the following properties:

(p1) $\mathcal{M}_{\epsilon} \rightarrow \mathcal{M}$ as $\epsilon \rightarrow 0$.

(p2) $\mathcal{M}_{\epsilon}$ has a singular point $X_{\epsilon}=\left(x_{\epsilon}, 0\right)$.

(p3) $\mathcal{M}_{\epsilon}$ has as a tangent flow at $X_{\epsilon}$ a self-similarly shrinking cylinder $\mathbf{S}^{k} \times \mathbf{R}^{n-k-1}$ for some $k=k_{\epsilon} \leq n-2$.

(p4) $X_{\epsilon} \rightarrow(0,0)$ as $\epsilon \rightarrow 0$.

We now explain why existence of such flows $\mathcal{M}_{\epsilon}$ implies the theorem. Let $d_{k}$ be the Gaussian density of a self-similarly shrinking cylinder $\mathbf{S}^{k} \times \mathbf{R}^{n-k-1}$. (Here $\mathbf{S}^{k}=\partial \mathbf{B}^{k+1}$ is the unit $k$-sphere in $\mathbf{R}^{k+1}$.) This Gaussian density is 
independent of $n$, and hence is also the Gaussian density of a shrinking $k$-sphere in $\mathbf{R}^{k+1}$. Indeed,

$$
\begin{aligned}
d_{k} & =\left(\frac{k}{2 \pi e}\right)^{k / 2} \sigma_{k} \\
& =2 \sqrt{\pi}\left(\frac{k}{2 e}\right)^{k / 2}\left(\frac{1}{\Gamma\left(\frac{k+1}{2}\right)}\right)
\end{aligned}
$$

(where $\sigma_{k}$ is the area of a $k$-dimensional sphere of radius 1 ), from which it follows that

$$
d_{1}>d_{2}>d_{3}>\ldots \text { and } \lim _{k \rightarrow \infty} d_{k}=\sqrt{2} .
$$

(See [Sto94, Appendix A] for proofs of these facts about $d_{k}$.) Thus

$$
\Theta\left(\mathcal{M}_{\epsilon}, X_{\epsilon}\right)=d_{k(\epsilon)} \geq d_{n-2},
$$

so

$$
\Theta(C, 0)=\Theta\left(\mathcal{M}_{0},(0,0)\right) \geq \limsup _{\epsilon \rightarrow 0} \Theta\left(\mathcal{M}_{\epsilon}, X_{\epsilon}\right) \geq d_{n-2}>\sqrt{2},
$$

as desired. Hence to prove the theorem, it suffices to construct mean curvature flows $\mathcal{M}_{\epsilon}$ with properties (p1) - (p4).

Since $C$ is an area-minimizing hypercone, it is one leaf of a foliation of $\mathbf{R}^{n}$ by area-minimizing hypersurfaces [HS85]. The foliation is of course singular at the origin, but it is smooth everywhere else. Each leaf other than $C$ is a radial graph over one of the components of $(\partial \mathbf{B}) \backslash C$. Furthermore, the foliation is invariant under positive dilations about the origin. We can conveniently represent the leaves as level sets of a function $u: \mathbf{R}^{n} \rightarrow \mathbf{R}$ as follows. Let $U^{+}$and $U^{-}$be the two connected components of $\mathbf{R}^{n} \backslash C$. By hypothesis, at least one of $U^{+}$and $U^{-}$is non-contractible. We choose the labeling so that $U^{-}$is non-contractible. We let $u(x)=0$ for points $x \in C$. For points $x \in \mathbf{R}^{n} \backslash C$, we let $u(x)$ be plus or minus the distance from the origin to the leaf through $x$ according to whether $x \in U^{+}$or $x \in U^{-}$. Note that

$$
u(\lambda x)=\lambda u(x) \text { for } \lambda>0
$$

since the foliation is invariant under positive dilations.

Let

$$
L_{v}:=u^{-1}(v) \cap \mathbf{B}
$$


be the portion of the leaf $u^{-1}(v)$ inside the unit ball $\mathbf{B}$.

Since the leaf $u^{-1}(v)$ converges smoothly (away from the origin) to $C$ as $v \rightarrow 0$, it follows that there is a $\delta>0$ such that the leaf $u^{-1}(v)$ intersects the unit sphere $\partial \mathbf{B}$ transversely provided $|v| \leq \delta$. We now construct the mean curvature flow $\mathcal{M}_{\epsilon}$ for each $\epsilon$ with $0<\epsilon \leq \delta$.

Our mean curvature flow $\mathcal{M}_{\epsilon}$ will be a flow of a surface with boundary. We first describe the motion of the boundary. Let $\tau: \mathbf{R} \rightarrow[-1,1)$ be a smooth increasing function such that

1. $\tau(t)=-1$ for $t \leq 0$,

2. $\tau^{\prime}(t)>0$ for $t>0$, and

3. $\tau(t) \rightarrow 1$ as $t \rightarrow \infty$.

For $t \in \mathbf{R}$, let

$$
\Gamma_{\epsilon}(t)=\partial L_{\epsilon \tau(t)} .
$$

Thus for $t \leq 0$, the boundary $\Gamma_{\epsilon}(t)$ is $\partial L_{-\epsilon}$. As $t \rightarrow \infty, \Gamma_{\epsilon}(t)$ converges smoothly to $\partial L_{\epsilon}$. Note that $t \mapsto \Gamma(t)$ is a smooth isotopy in $\partial \mathbf{B}$.

Now let

$$
\mathcal{M}_{\epsilon}: t \in[0, \infty) \mapsto M_{\epsilon}(t)
$$

be the mean curvature flow (of surfaces with boundary) such that:

1. Initially (i.e., at time $t=0$ ), the surface is $L_{-\epsilon}$.

2. At each time $t \geq 0$, the boundary of the surface is $\Gamma_{\epsilon}(t)$.

(For existence of the flow, see theorem 4 in the appendix, which is proved by elliptic regularization as in [Ilm94]. However, for the proof of theorem 1, it would suffice to consider the flow $t \in[0, T) \mapsto M_{\epsilon}(t)$ up to the first singular time.)

Note that since the surface is initially minimal, and since the boundary is always moving to one side of the surface, it follows (by the strong maximum principle) that for $t>0$, the surface $M_{\epsilon}(t)$ has nowhere vanishing mean curvature. (See assertion 2 in theorem 4 in the appendix.)

We extend the flow to all time by setting

$$
M_{\epsilon}(t)=M_{\epsilon}(0)=L_{-\epsilon} \quad \text { for } t \leq 0 .
$$

By the maximum principle (or by construction, if one uses elliptic regularization to construct the flow), the surfaces $M_{\epsilon}(t)$ all lie in the region where $-\epsilon \leq u(x) \leq \epsilon$ :

$$
M_{\epsilon}(t) \subset \mathbf{B} \cap\{x:-\epsilon \leq u(x) \leq \epsilon\} .
$$


(To see this using the maximum principle, note that the quantity

$$
\sup \left\{|x|: x \in M_{\epsilon}(t)\right\}
$$

is a decreasing function of $t$, that

$$
\inf \left\{u(x): x \in M_{\epsilon}(t)\right\}
$$

is an increasing function of $t$, and that the quantity

$$
\sup \left\{u(x): x \in M_{\epsilon}(t)\right\}
$$

is a decreasing function of $t$ on any interval on which it is $>\epsilon$.)

As $\epsilon \rightarrow 0$, any subsequence of the flows $\mathcal{M}_{\epsilon}$ has a further subsequence that converges to a Brakke flow $\mathcal{M}_{0}$. By $(6)$, for each time $t, M_{0}(t)$ is a varifold supported in $C \cap \mathbf{B}$. In fact, for $t \leq 0, M_{0}(t)$ is the multiplicity 1 varifold associated to $C \cap \mathbf{B}$. Consequently, either this holds for all $t$, or else there is some time $T$ such that the $M_{0}(t)$ vanishes at time $T$. (That is, such that $M_{0}(t)$ is the zero varifold for $t>T$.) But such vanishing is impossible because at all times, $M_{\epsilon}(t)$ supports an integral current whose boundary is an integral current with support $\Gamma_{\epsilon}(t)$.

By the local regularity theory in [Whi05], for all sufficiently small $\epsilon$, the flows $\mathcal{M}_{\epsilon}$ are regular away from the line $0 \times \mathbf{R}$. That is, if $\epsilon(i) \rightarrow 0$ and if $\left(x_{\epsilon(i)}, t_{\epsilon(i)}\right)$ is a singular point of the flow $\mathcal{M}_{\epsilon(i)}$, then $x_{\epsilon(i)} \rightarrow 0$. In particular, there are no singularities at the boundary.

Claim 1. For sufficiently large $t$ (depending on $\epsilon$ ) the surfaces $M_{\epsilon}(t)$ are smooth and converge smoothly to $L_{\epsilon}$.

It suffices to prove weak convergence to $L_{\epsilon}$, since smooth convergence then follows from the local regularity theory [Whi05]. There are many ways to prove weak convergence. For example, by assertion 5 of theorem 4 in the appendix, the surface $M_{\epsilon}(t)$ converges as $t \rightarrow \infty$ to a minimal hypersurface $H \subset \mathbf{B}$ with boundary $\partial L_{\epsilon}$ and with a singular set $Z$ of Hausdorff dimension at most $n-8$. The convergence is smooth away from $Z$. If the claim were not true, then $v:=\min \{u(x): x \in H\}$ would be strictly less than $\epsilon$. Then for $s<v$ and sufficiently close to $v$, the shortest distance from the leaf $L_{s}$ to $H$ is realized by points $p$ and $q$ where $p$ is in the interior of $L_{s}$ and $q$ is in $H \backslash \partial H$. It follows that immediately that the tangent cones to $L_{s}$ at $p$ and to $H$ at $q$ lie in halfspaces and are therefore planes. Consequently, $p$ and $q$ are regular points of $L_{s}$ and of $H$, and therefore we get a contradiction to the strong maximum principle.) This completes the proof of claim 1. 
Claim 2. If $\epsilon$ is sufficiently small, the flow $\mathcal{M}_{\epsilon}$ must have a singularity.

For suppose not. By hypothesis, the component $U^{-}$of $\mathbf{R}^{n} \backslash C$ is not contractible. Thus at least one of its homotopy groups, say the $k^{\text {th }}$, is nontrivial. Hence there is a map $f: \mathbf{S}^{k} \rightarrow U^{-}$from the $k$-sphere to $U^{-}$that is homotopically nontrivial in $U^{-}$. By dilating, we may assume that $f\left(\mathbf{S}^{k}\right)$ lies in $U^{-} \cap \mathbf{B}$. We assume that $\epsilon$ is small enough that $u<-\epsilon$ on $f\left(\mathbf{S}^{k}\right)$. (In other words, $f\left(\mathbf{S}^{k}\right)$ and the origin lie on opposite sides of $L_{-\epsilon}$.)

On the other hand, $f$ is homotopically trivial in $\mathbf{B} \cap \overline{U^{-}}$by the homotopy

$$
\begin{aligned}
& H: \mathbf{S}^{k} \times[0,1] \rightarrow \mathbf{B} \cap\{u \leq a\}, \\
& H(x, v)=v f(x) .
\end{aligned}
$$

Now if the flow $\mathcal{M}_{\epsilon}$ had no singularities, then it would provide a smooth isotopy from $L_{-\epsilon}$ to $L_{\epsilon}$. Using the isotopy, we could push

$$
H\left(\mathbf{S}^{k} \times[0,1]\right)
$$

into $U^{-}$, leaving $f\left(\mathbf{S}^{k}\right)=H\left(\mathbf{S}^{k} \times\{1\}\right)$ fixed, which means that $f$ would be homotopically trivial in $U^{-}$, contradicting the choice of $f$. The contradiction proves that the flow $\mathcal{M}_{\epsilon}$ has a singularity.

Claim 3. If $\epsilon$ is sufficiently small, the flow $\mathcal{M}_{\epsilon}$ must have a singularity with a self-similarly shrinking $\mathbf{S}^{j} \times \mathbf{R}^{n-j-1}$ as a tangent flow.

Let $X_{\epsilon}=\left(x_{\epsilon}, t_{\epsilon}\right)$ be a singularity of the flow. For this theorem, we may as well choose $t_{\epsilon}$ to be the first time at which a singularity occurs. (In the proof of Theorem 2 below, we will make a different choice.) Let $\mathcal{T}_{\epsilon}$ be a tangent flow to $\mathcal{M}_{\epsilon}$ at $\left(x_{\epsilon}, t_{\epsilon}\right)$. Then $\mathcal{T}_{\epsilon}$ is a self-similarly shrinking cylinder $\mathbf{S}^{j} \times \mathbf{R}^{n-j-1}$ for some $j$ with $1 \leq j \leq n-1$ by theorem 5 in the appendix ${ }^{1}$. (If $t_{\epsilon}$ is the first singular time, this also follows from [Whi03, Theorem 1].)

This completes the proof of claim 3 , but we remark that in fact $j \neq n-1$, and thus that $j \leq n-2$. To see this, note that for $t<t_{\epsilon}$, the surfaces $M_{\epsilon}(t)$ are diffeomorphic to $M_{\epsilon}(0)$ and hence are connected manifolds with nonempty boundary. Now if $j$ were equal to $n-1$, that is, if a tangent flow $\mathcal{T}_{\epsilon}$ at $\left(x_{\epsilon}, t_{\epsilon}\right)$ were a shrinking sphere, then just before the singularity, $M_{\epsilon}(t)$ would have a compact component diffeomorphic to a sphere, a contradiction. Thus $j \leq n-2$.

Now we have proved that $\mathcal{M}_{\epsilon}$ has all the desired properties, except that we do not know that $\left(x_{\epsilon}, t_{\epsilon}\right) \rightarrow(0,0)$. Thus we modify the flow by translating

\footnotetext{
${ }^{1}$ By claim 1 , the hypothesis of theorem 5 holds.
} 
in time by $-t_{\epsilon}$. The new flow $\mathcal{M}_{\epsilon}^{\prime}$ has a shrinking-cylinder type singularity at $X_{\epsilon}^{\prime}=\left(x_{\epsilon}, 0\right)$, and $X_{\epsilon}^{\prime} \rightarrow(0,0)$. Furthermore, as $\epsilon \rightarrow 0, \mathcal{M}_{\epsilon}^{\prime}$ converges to $\mathcal{M}$ for the same reason that $\mathcal{M}_{\epsilon}$ converges to $\mathcal{M}$. We have proved that the flow $\mathcal{M}_{\epsilon}^{\prime}$ has all the properties (p1) - (p4), completing the proof of the theorem.

We have actually proved a little more than was asserted in the theorem:

Theorem $\mathbf{1}^{*}$. Let $C \in \mathbf{R}^{n}$ be an area-minimizing hypercone with an isolated singularity at the origin, and suppose that $C$ is topologically nontrivial (as in Theorem 1). Then the density of $C$ at the origin is greater than or equal to $d_{n-2}$, the Gaussian density of a shrinking $(n-2)$-sphere in $\mathbf{R}^{n-1}$.

For a better bound, see the corollary to theorem 2 below.

\section{Bounding density in terms of topology}

Theorem 2. Suppose that $C \subset \mathbf{R}^{n}$ is an area-minimizing hypercone with an isolated singularity at the origin. Suppose also that one of the components of $\mathbf{R}^{n} \backslash C$ has nontrivial $k^{\text {th }}$ homotopy group. Then

$$
\Theta(C) \geq d_{k}
$$

where

$$
d_{k}=\left(\frac{k}{2 \pi e}\right)^{k / 2} \sigma_{k}
$$

is the Gaussian density of a shrinking k-sphere in $\mathbf{R}^{k+1}$.

Here $\sigma_{k}$ is the area of the unit sphere in $\mathbf{R}^{k+1}$.

Proof. For $0<\epsilon \leq \delta$, let $\mathcal{M}_{\epsilon}$ be the flow constructed in the proof of theorem 1 . We may suppose that the $k^{\text {th }}$ homotopy group of $U^{-}$is non-trivial. Thus there is a continuous map $f: \mathbf{S}^{k} \rightarrow U^{-}$that is homotopically nontrivial in $U^{-}$.

By dilating, we may assume that $f\left(\mathbf{S}^{k}\right)$ lies in $\mathbf{B} \cap U^{-}$. We will assume from now on that $\epsilon$ is sufficiently small that $u<-\epsilon$ on $f\left(\mathbf{S}^{k}\right)$.

Let $W(0)=\{x \in \mathbf{B}: u(x)<\epsilon\}$. For $0<t \leq \infty$, let

$$
W(t)=W(0) \cup\left(\bigcup_{T<t} M_{\epsilon}(T)\right) .
$$


If we think of the $M_{\epsilon}(t)$ as moving forward, then $W(t)$ is the portion of $\mathbf{B}$ that lies behind $M_{\epsilon}(t)$. Note that

$$
W(\infty)=\{x \in \mathbf{B}: u(x)<\epsilon\}
$$

Note also that $W(\infty)$ is star-shaped (by $(5)$ ), so $f$ is homotopically trivial in $W(\infty)$.

Thus $f$ is homotopically trivial in $W(\infty)$ but not in $W(0)$ (since $W(0) \subset$ $U^{-}$). By theorem 6 (together with theorem 5) in the appendix, this implies that there is a point $X_{\epsilon}=\left(x_{\epsilon}, t_{\epsilon}\right)$ at which the tangent flow to $\mathcal{M}_{\epsilon}$ is a shrinking $\mathbf{S}^{j} \times \mathbf{R}^{n-j-1}$ for some $j \leq k$. Thus

$$
\Theta\left(\mathcal{M}_{\epsilon}, X_{\epsilon}\right)=d_{j} \geq d_{k}
$$

Now exactly as in the proof of theorem 1, this implies that

$$
\Theta(C, 0) \geq \limsup _{\epsilon \rightarrow 0} \Theta\left(\mathcal{M}_{\epsilon}, X_{\epsilon}\right) \geq d_{k}
$$

Corollary. Let $C$ be an area-minimizing hypercone in $\mathbf{R}^{n}$ with an isolated singularity at the origin. Suppose that at least one of the components of $\mathbf{R}^{n} \backslash C$ is not contractible. Then

$$
\Theta(C) \geq d_{[(n-2) / 2]}
$$

where $[(n-2) / 2]$ is the greatest integer less than or equal to $(n-2) / 2$.

This improves on the bound $\Theta(C) \geq d_{n-2}$ given by theorem $1^{*}$.

Proof. Let $U^{-}$and $U^{+}$be the two components of $\mathbf{R}^{n} \backslash C$, and let $V^{-}$and $V^{+}$be the corresponding components of $(\partial \mathbf{B}) \backslash C$. Note that $U^{-}$and $U^{+}$ are homemorphic to $V^{-} \times \mathbf{R}$ and $V^{+} \times \mathbf{R}$ and thus are homotopy equivalent to $V^{-}$and $V^{+}$.

We may suppose that $U^{-}$is not contractible, so that one of its homotopy groups is not trivial. Let $k$ be the smallest integer $\geq 1$ such that $\pi_{k}\left(U^{-}\right)$is nontrivial.

If $k=1$, then $\Theta(C, \mathbf{O}) \geq d_{1} \geq d_{[(n-1) / 2]}$ and we are done. Thus we may assume that $k>1$. In particular, $U^{-}$is simply connected, so by the Hurewicz Theorem, the homology group $H_{k}\left(U^{-}\right)$is nontrivial. Thus

$$
\begin{aligned}
0 & \neq H_{k}\left(U^{-}\right) \\
& =H_{k}\left(V^{-}\right)
\end{aligned}
$$




$$
\begin{aligned}
& =H_{k}\left(\overline{V^{-}}\right) \\
& =H^{n-k-2}\left(V^{+}\right) \quad \text { (by Alexander duality) } \\
& =H^{n-k-2}\left(U^{+}\right)
\end{aligned}
$$

By the universal coefficients theorem, the nontriviality of $H^{n-k-2}\left(U^{+}\right)$implies that $H_{n-k-3}\left(U^{+}\right)$and $H_{n-k-2}\left(U^{+}\right)$cannot both be trivial. The Hurewicz theorem then implies that $\pi_{j}\left(U^{+}\right)$is nontrivial for some $j \leq n-k-2$.

Thus $j+k \leq n-2$, so if $p$ is the smaller of $j$ and $k$, then $p \leq[(n-2) / 2]$. Hence by theorem 2 ,

$$
\Theta(C, 0) \geq d_{p} \geq d_{[(n-2) / 2]} .
$$

\section{Examples}

For positive integers $m$ and $n$, let $C_{m, n}$ be the Simons' cone

$$
C_{m, n}=\left\{(x, y) \in \mathbf{R}^{m+1} \times \mathbf{R}^{n+1}=\mathbf{R}^{m+n+2}: n|x|^{2}=m|y|^{2}\right\} .
$$

This cone is minimal, and it is area-minimizing if and only either (i) $m+n \geq$ 6 or (ii) $m+n=6$ and neither $m$ nor $n$ is equal to 1 (see [Law91]).

The cone divides the unit sphere into two components, one of which is the product of an $m$-sphere and an $n$-ball. Therefore the corresponding component of the complement of $C$ has nontrivial $m^{\text {th }}$ homotopy group, so according to theorem 2 ,

$$
\Theta\left(C_{m, n}\right) \geq d_{m} .
$$

Thus the following theorem shows that the constant $d_{m}$ in theorem 2 cannot be replaced by any larger constant.

Theorem 3. $\lim _{n \rightarrow \infty} \Theta\left(C_{m, n}\right)=d_{m}$.

Proof. The intersection $\Gamma_{m, n}$ of $C_{m, n}$ with the unit sphere is the Cartesian product of the $m$-sphere of radius $\sqrt{\frac{m}{m+n}}$ and the $n$-sphere of radius $\sqrt{\frac{n}{m+n}}$.

Thus

$$
\begin{aligned}
\operatorname{area}\left(\Gamma_{m, n}\right) & =\sigma_{m}\left(\sqrt{\frac{m}{m+n}}\right)^{m} \cdot \sigma_{n}\left(\sqrt{\frac{n}{m+n}}\right)^{n} \\
& =\sigma_{m} \sigma_{n}\left(\frac{m}{m+n}\right)^{m / 2}\left(\frac{n}{m+n}\right)^{n / 2}
\end{aligned}
$$


where $\sigma_{k}$ is the area of a $k$-dimensional sphere of radius $k$. To get the density of the cone, we divide the area of $\Gamma_{m, n}$ by the area $\sigma_{m+n}$ of a unit sphere of the same dimension:

$$
\Theta\left(C_{m, n}\right)=\frac{\sigma_{m} \sigma_{n}}{\sigma_{m+n}}\left(\frac{m}{m+n}\right)^{m / 2}\left(\frac{n}{m+n}\right)^{n / 2} .
$$

As usual, we write $A \sim B$ to mean $\lim _{n \rightarrow \infty}(A / B)=1$. Note that

$$
\left(\frac{m}{m+n}\right)^{m / 2}=\left(\frac{m}{n}\right)^{m / 2}\left(\frac{n}{m+n}\right)^{m / 2} \sim\left(\frac{m}{n}\right)^{m / 2}
$$

and

$$
\left(\frac{n}{m+n}\right)^{n / 2}=\left(1+\frac{m}{n}\right)^{-n / 2} \rightarrow e^{-m / 2}
$$

so

$$
\Theta\left(C_{m, n}\right) \sim \sigma_{m} \frac{\sigma_{n}}{\sigma_{m+n}}\left(\frac{m}{n e}\right)^{m / 2} .
$$

Now

$$
\sigma_{k}=(k+1) \omega_{k+1}=(k+1) \frac{\pi^{(k+1) / 2}}{\Gamma\left(\frac{k+3}{2}\right)},
$$

where $\omega_{k+1}$ is the volume of the unit ball in $\mathbf{R}^{k+1}$.

Thus

$$
\frac{\sigma_{n}}{\sigma_{m+n}}=\frac{n+1}{m+n+1} \cdot \frac{\omega_{n+1}}{\omega_{m+n+1}} \sim \frac{\omega_{m+1}}{\omega_{m+n+1}}=\frac{1}{\pi^{m / 2}} \cdot \frac{\Gamma\left(\frac{m+n+3}{2}\right)}{\Gamma\left(\frac{n+3}{2}\right)} .
$$

From Stirling's approximation

$$
\Gamma(z) \sim \sqrt{\frac{2 \pi}{z}}\left(\frac{z}{e}\right)^{z},
$$

one checks that

$$
\frac{\Gamma(z+a)}{\Gamma(z)} \sim z^{a}
$$

as $z \rightarrow \infty$ with $a$ fixed. Thus (10) becomes

$$
\frac{\sigma_{n}}{\sigma_{m+n}} \sim \frac{1}{\pi^{m / 2}} \cdot\left(\frac{n+3}{2}\right)^{m / 2} \sim\left(\frac{n}{2 \pi}\right)^{m / 2} .
$$


Combining this with (8) gives

$$
\Theta\left(C_{m, n}\right) \sim \sigma_{m}\left(\frac{m}{2 \pi e}\right)^{m / 2},
$$

which is precisely $d_{m}$ (see (3)).

In a similar manner, one can use (7) and Stirling's approximation to check that $\lim _{n \rightarrow \infty} \Theta\left(C_{n, n}\right)=\sqrt{2}$, which shows that $\sqrt{2}$ is the best possible constant in theorem 1 . Alternatively, one can see that $\sqrt{2}$ is optimal because

$$
\lim _{n \rightarrow \infty} \lim _{m \rightarrow \infty} \Theta\left(C_{m, n}\right)=\lim _{n \rightarrow \infty} d_{n}=\sqrt{2} .
$$

by (11) and (4).

Although the bounds $\sqrt{2}$ and $d_{n}$ in theorems 1 and 2 are the best constants independent of dimension, they are not optimal if one considers cones of a given dimension $N$. However, the bound from theorem 2 is surprisingly good even when $N$ is small. (Small here means close to 7, the smallest dimension $N$ for which there exist $N$-dimensional singular area minimizing hypersones.) For example, consider the 8-dimensional cone $C_{1,6}$, the simplest known area-minimizing hypercone whose complement contains a component that is not simply connected. According to theorem 2, its density is greater than $d_{1}=1.520$. In fact, its density (which can be calculated from (7) and (9)) is 1.523 . Thus the density is only $0.2 \%$ higher than the lower bound from theorem 2. By contrast, its density is about $8 \%$ higher than $\sqrt{2}=1.414$, the lower bound from theorem 1 .

\section{Open problems}

1. Prove that in Theorem 1, we can drop one or more of the following hypotheses: that $C$ be minimizing, that $C$ be topologically nontrivial, and that $C$ have an isolated singularity.

2. Prove that in Theorem 2, we can drop one or both of the hypotheses that $C$ be area-minimizing and that $C$ have an isolated singularity.

3. (Conjectured by Solomon.) For $m \geq 1$, prove that the Simon's cone $C_{m, m}$ is the $(2 m+1)$-dimensional minimal (or area-minimizing) hypercone of least possible density. For $m=1$, one has to exclude cones with soap-film-like triple junctions, since the density of 3 half planes meeting along a common edge is $3 / 2$, which is less than $\Theta\left(C_{1,1}\right)$. However, in higher dimensions this exclusion is not necessary since $\Theta\left(C_{m, m}\right)<3 / 2$ for $m>1$. 
4. (Conjectured by Solomon.) Prove that the cone $C_{m, m+1}$ is the $(2 m+2)$ dimensional minimal (or area-minimizing) hypercone of least possible density.

5. Prove that $C_{m, n}$ has the least possible density among all $(m+n+1)$ dimensional minimal (or area-minimizing) hypercones $C$ such that at least one of the components of the complement has nontrivial $m^{\text {th }}$ homotopy group.

6. Prove lower density bounds for minimal or for area-minimizing cones of codimension $>1$.

7. Find a conceptual explanation for why the bounds in this paper are sharp. The authors find it mysterious that the method of proof here gives such good bounds.

\section{Appendix A. Mean curvature flow}

In this section, we state the fundamental results about existence and singularity structure for a mean-convex hypersurface $M_{t}$ moving by mean curvature in an ambient space $N$, where the prescribed Dirichlet data consist of the initial surface $\Sigma=M_{0}$ and the motion $t \mapsto \Gamma_{t}=\partial M_{t}$ of the boundary. Throughout this section, we make the following assumptions:

(1) $N$ is a smooth Riemannian $n$-manifold with smooth boundary. At each boundary point, the mean curvature is a nonnegative multiple of the inward unit normal. (In this paper, $N$ is the unit ball in $\mathbf{R}^{n}$.)

(2) $t \in[0, \infty) \mapsto \Gamma_{t}$ is a smooth, 1-parameter family of smoothly embedded, closed $(n-2)$-manifolds in $\partial N$. As $t \rightarrow \infty$, the $\Gamma_{t}$ converge smoothly to a smoothly embedded $(n-2)$ manifold $\Gamma_{\infty}$.

(3) The family $t \mapsto \Gamma_{t}$ is monotonic is the following sense: $\Gamma_{t}$ is boundary of a region $U_{t} \subset \partial N$ where $U(t) \supset U\left(t^{\prime}\right)$ for $t \leq t^{\prime}$.

(4) The initial surface $\Sigma$ is a smoothly embedded, compact $(n-1)$-manifold in $N$ such that

$$
\partial \Sigma=\Gamma_{0}
$$

and such that $\Sigma$ and $\partial N$ are nowhere tangent ${ }^{2}$.

(5) The surfaces $\Sigma$ and $U_{0}$ together bound a region $\Omega$ in $N$, and at each point of $\Sigma$, the mean curvature vector is a nonnegative multiple of the unit normal pointing into $\Omega$.

\footnotetext{
${ }^{2}$ Actually, it suffices to assume that $\Sigma$ and $\overline{U_{0}}$ are nowhere tangent. Indeed, the hypothesis is stated that way in [Whi13b]. This allows, for example, the initial surface $\Sigma$ to be $(\partial N) \backslash U_{0}$.
} 
(6) If $\Sigma$ is a minimal surface, then $\Gamma_{t} \neq \Gamma_{0}$ for $t>0$. (This is to guarantee that surface $M_{t}$ starts moving as soon as $t$ is positive.)

Theorem 4. Under the hypotheses above, there is a unique weak solution $t \in[0, \infty) \mapsto M_{t}$ of mean curvature flow such that $M_{0}=\Sigma$ and such that $\partial M_{t}=\Gamma_{t}$ for all $t$. The surfaces $M_{t} \backslash \Gamma_{t}$ are disjoint (for distinct values of $t$ ), and the time-of-arrival function

$$
\begin{aligned}
& \tau: \bar{\Omega} \backslash \partial N \rightarrow[0, \infty] \\
& \tau(x)= \begin{cases}t & \text { if } x \in M_{t} \\
\infty & \text { if } x \notin \cup_{0 \leq t<\infty} M_{t}\end{cases}
\end{aligned}
$$

is a continuous function. Each $M_{t}$ is rectifiable, and the multiplicity-one varifolds associated to the $M_{t}$ form a Brakke flow.

Furthermore, there is a compact subset (the singular set) $Q$ of $\Omega$ with the following properties:

(1) The set $Q$ has Hausdorff dimension at most $(n-2)$, and the spacetime singular set has parabolic Hausdorff dimension at most $(n-2)$.

(2) Each $M_{t} \backslash Q$ with $t \in(0, \infty)$ is a smooth, properly embedded submanifold of $N \backslash Q$ with boundary $\Gamma_{t}$, and the mean curvature of $M_{t} \backslash\left(Q \cup \Gamma_{t}\right)$ is everywhere positive.

(3) If $t>0$ and $t(i) \rightarrow t$, then $M_{t(i)}$ converges smoothly to $M_{t}$ away from $M_{t} \cap Q$.

(4) If $t(i) \rightarrow 0$, then $M_{t(i)}$ converges in $C^{1, \alpha}$ (for every $\left.\alpha \in(0,1)\right)$ to $M_{0}$, and the convergence is smooth except at the boundary.

(5) The surface $M_{t}$ converges as $t \rightarrow \infty$ to a minimal variety $M_{\infty}$, and the convergence is smooth away from the singular set $Q \cap M_{\infty}$, which has Hausdorff dimension at most $n-8$.

See [Whi13b, theorem 4] for the proof.

Of course "there is a unique weak solution" is somewhat informal. However, uniqueness is not needed in this paper. (The precise uniqueness statement is: the flow $t \in[0, \infty) \mapsto M_{t}$ is the level set flow generated by $\Sigma$. Note that this flow is non-fattening since by assertion 2 of the theorem, $M_{t}$ contains no non-empty open subset of $N$. See [Whi95] for level set flow of surfaces with boundary.)

If $M$ is an $(n-1)$-dimensional hypersurface and $x \in M$ is a point where the mean curvature is nonzero, let

$$
\Phi(M, x)=\frac{\kappa_{1}}{h}
$$


where $\kappa_{1} \leq \kappa_{2} \leq \cdots \leq \kappa_{n-1}$ are the principal curvatures of $M$ at $x$ and

$$
h=\sum_{i} \kappa_{i}>0
$$

is the mean curvature of $M$ at $x$. (One can think of $\Phi(M, x)$ as a dimensionless measure of convexity. In particular, $\Phi(M, x) \geq 0$ if and only if $M$ is convex to second order at $x$.) We say a singularity $(x, t)$ with $x \in M_{t}$ has convex type provided:

1. Each tangent flow at $(x, t)$ is a self-similarly shrinking $\mathbf{S}^{k} \times \mathbf{R}^{n-k-1}$ for some $k \geq 1$.

2. If $x_{i} \in M_{t_{i}}$ is a sequence of regular points converging to $(x, t)$, then

$$
\liminf _{i \rightarrow \infty} \Phi\left(M_{t_{i}}, x_{i}\right) \geq 0
$$

It seems likely that all of the finite-time singularities of the flow in theorem 4 must have convex type. In some situations, it is known that the singularities have convex type:

Theorem 5. Let $t \mapsto M_{t}$ be as in theorem 4. Suppose that either

1. $n<8$, or

2. $N \subset \mathbf{R}^{n}$ (with the Euclidean metric) and $M_{\infty}$ is smooth.

Then all of the finite-time singularities of the flow have convex type.

See [Whi13b, theorems 5 and 6] for the proof. (It may seem peculiar to require any hypothesis on $M_{\infty}$ for a parabolic problem. The hypothesis arises because the proof is by elliptic regularization.)

Now extend the time-of-arrival function $\tau$ in theorem 4 to all of $N \backslash \partial N$ by setting $\tau(x)=-\infty$ for $x \notin \bar{\Omega}$. Of course $\tau$ will now be discontinuous along $M_{0}$. Let $W(t)=\{x: \tau(x)<t\}$. If one thinks of $M_{t}$ as moving forward, then for $t \geq 0$, the region $W(t)$ is the region in the interior of $N$ that lies behind the surface $M_{t}$. Note that the topology of $W(t)$ can change only when there are singularities in the flow. Not surprisingly, the way that the topology changes gives information about those singularities:

Theorem 6. Let $t \mapsto M_{t}$ be as in theorem 4, and suppose that all the finitetime singularities have convex type. Let $0 \leq t<t^{\prime} \leq \infty$ and suppose that $f: \mathbf{S}^{k} \rightarrow W(t)$ is homotopically trivial in $W\left(t^{\prime}\right)$ but not in $W(t)$. Then there is a singularity $\left(x_{0}, t_{0}\right)$ with $t \leq t_{0}<t^{\prime}$ that has as a tangent flow a shrinking $\mathbf{S}^{j} \times \mathbf{R}^{n-j-1}$ for some $j \leq k$. 
See [Whi13a, theorem 5.3] for the proof. (The theorem there is stated under the additional hypotheses in theorem 5 above, but as explained in the paragraph following [Whi13a, theorem 5.3], those hypotheses are used only to guarantee that the finite-time singularities have convex type.)

The Gaussian density $d_{k}$ of a shrinking $\mathbf{S}^{k} \times \mathbf{R}^{n-k-1}$ is a strictly decreasing function of $k$. (See (4) in section 2.) Thus theorem 6 implies the following: to kill an element of the $k^{\text {th }}$ homotopy of $W(t)$ requires a singularity of Gaussian density $\geq d_{k}$.

\section{References}

[HS85] Robert Hardt and Leon Simon, Area minimizing hypersurfaces with isolated singularities, J. Reine Angew. Math. 362 (1985), 102-129. MR809969 (87f:49052)

[Hsi83a] Wu-Yi Hsiang, Minimal cones and the spherical Bernstein problem. I, Ann. of Math. (2) 118 (1983), no. 1, 6173. MR707161 (85e:53080a)

[Hsi83b] - Minimal cones and the spherical Bernstein problem. II, Invent. Math. 74 (1983), no. 3, 351-369. MR724010 (85e:53080b)

[HS86] Wu-Yi Hsiang and Ivan Sterling, Minimal cones and the spherical Bernstein problem. III, Invent. Math. 85 (1986), no. 2, 223247. MR846927 (87k:53139)

[Ilm94] Tom Ilmanen, Elliptic regularization and partial regularity for motion by mean curvature, Mem. Amer. Math. Soc. 108 (1994), no. 520, x+90. MR1196160 (95d:49060)

[Law91] Gary R. Lawlor, A sufficient criterion for a cone to be areaminimizing, Mem. Amer. Math. Soc. 91 (1991), no. 446, vi+111. MR1073951 (92d:49079)

[Maz61] Barry Mazur, A note on some contractible 4-manifolds, Ann. of Math. (2) 73 (1961), 221-228. MR0125574 (23 \#A2873)

[Sto94] Andrew Stone, A density function and the structure of singularities of the mean curvature flow, Calc. Var. Partial Differential Equations 2 (1994), no. 4, 443-480. MR1383918 (97c:58030)

[Sto96] _ A boundary regularity theorem for mean curvature flow, J. Differential Geom. 44 (1996), no. 2, 371434. MR1425580 (97m:58050) 
[Whi95] Brian White, The topology of hypersurfaces moving by mean curvature, Comm. Anal. Geom. 3 (1995), no. 1-2, $317-$ 333. MR1362655 (96k:58051)

[Whi97] _ Stratification of minimal surfaces, mean curvature flows, and harmonic maps, J. Reine Angew. Math. 488 (1997), 135. MR1465365 (99b:49038)

[Whi00] , The size of the singular set in mean curvature flow of mean-convex sets, J. Amer. Math. Soc. 13 (2000), no. 3, 665-695 (electronic). MR1758759 (2001j:53098)

[Whi03] - The nature of singularities in mean curvature flow of mean-convex sets, J. Amer. Math. Soc. 16 (2003), no. 1, 123-138 (electronic). MR1937202 (2003g:53121)

[Whi05] _ A local regularity theorem for mean curvature flow, Ann. of Math. (2) $\mathbf{1 6 1}$ (2005), no. 3, 14871519. MR2180405 (2006i:53100)

[Whi13a] _ Topological change in mean convex mean curvature flow, Invent. Math. 191 (2013), no. 3, 501-525. MR3020169

[Whi13b] _ Subsequent singularities in mean-convex mean curvature flow, Calc. Var. Partial Differential Equations (2013). To appear (preprint available on arXiv:1103.1469).

TOM ILMANEN

Departement MATHEMATIK

ETH ZENTRUM

RÄMISTRASSE 101 CH-8092

ZÜRICH

SWITZERLAND

E-mail address: ilmanen@math.ethz.ch

BRIAN WHITE

Department of Mathematics

STANFORD UNIVERSITY

STANFORD, CA 94305

USA

E-mail address: bcwhite@stanford.edu

ReCeIVed April 1, 2014 